\title{
A DIRECT GEOMETRIC PROOF OF THE LEFSCHETZ FIXED POINT FORMULAS
}

\author{
JOHN D. LAFFERTY, YU YANLIN, AND ZHANG WEIPING
}

\begin{abstract}
In this paper we prove the Lefschetz fixed point formulas of Atiyah, Singer, Segal, and Bott for isometries by using the direct geometric method initiated by Patodi.
\end{abstract}

\section{INTRODUCTION}

In this paper we present a very simple and direct geometric proof of the Lefschetz fixed point formulas by computing the equivariant index of the Dirac operator with respect to an isometry of the base manifold. The present work may be seen as a completion of the program initiated by Patodi in $[7,8]$, studying the local asymptotics of the heat kernel for the de Rham complex.

In recent years, several new proofs of the index theorem have been presented. Getzler [5] and, independently, Yu [9] evaluated the local index in terms of the asymptotics of the harmonic oscillator. Berline and Vergne [3] lifted the problem to the frame bundle, and evaluated the index in terms of the Jacobian of the exponential map. In a different direction, Bismut [4] expressed the trace of the Dirac heat kernel in terms of the local fluctuations of a Brownian motion, stochastically transporting frames along its sample paths. Each of these methods represents a heat equation approach.

The methods of $[3,4]$ were directly applicable to the situation where a group of isometries acts. In contrast, the evaluation of the local asymptotics of the $\hat{A}$ genus carried out in $[5,9]$ is expedited by the use of geodesic coordinates, i.e., calculating with respect to the moving frame obtained by parallelly translating along rays through the origin in a normal coordinate neighborhood. While this is the key to the simplicity of the method, it is not directly compatible with a calculation of the asymptotics in a neighborhood of the fixed-point submanifold of a group action.

In the following sections we complete the calculation of the equivariant index by extending the methods of $[8,9]$ to allow for the action of an isometry. After presenting notation and discussing the standard setup in $\S 2$, we discuss in $\S 3$ the particular moving frames necessary to the computation. Here it is seen that the

Received by the editors August 17, 1989 and, in revised form, November 8, 1989. Presented to the Conference on Topological Fixed Point Theory and Applications, held at the Nankai Institute of Mathematics, Tianjin, People's Republic of China, April 5-8, 1988.

1980 Mathematics Subject Classification (1985 Revision). Primary 58C30, 53B20; Secondary 57R15, 53A55. 
geodesic moving frame is related by an infinitesimal holonomy to the natural trivialization of the normal bundle. The final sections then evaluate the Clifford asymptotics of the local Lefschetz index by direct computation. The approach is completely elementary, and proceeds from first principles.

\section{Preliminaries}

Let $M$ be a $C^{\infty}$, compact, connected, and oriented Riemannian manifold, of even dimension $2 n$, with a fixed $\operatorname{Spin}(2 n)$ structure. There is thus a principal $\operatorname{Spin}(2 n)$-bundle $\operatorname{Spin}(M)$ for which $\operatorname{Spin}(M) \times_{\rho} S O(2 n)$ is the oriented orthonormal frame bundle $S O(M)$ of $M$, where $\rho: \operatorname{Spin}(2 n) \rightarrow S O(2 n)$ is the two-fold covering homomorphism. Let $\mathscr{S}_{+}$and $\mathscr{S}_{-}$be the spaces of positive and negative spinors. Then $\operatorname{Spin}(2 n)$ acts irreducibly on each of $\mathscr{S}_{+}$and $\mathscr{S}_{-}$, which are Clifford modules of dimension $2^{n-1}$. If $\left\{e_{i}\right\}$ denotes the set of generators for the Clifford algebra $\mathscr{C}_{2 n}=\operatorname{End}\left(\mathscr{S}_{+} \oplus \mathscr{S}_{-}\right)$then $i^{n} e_{1} e_{2} \cdots e_{2 n}$ acts as $\pm I$ on $\mathscr{S}_{ \pm}$. Define vector bundles $E_{+}$and $E_{-}$by setting $E_{ \pm}=\operatorname{Spin}(M) \times_{\operatorname{Spin}(2 n)} \mathscr{S}_{ \pm}$and Dirac operators $D_{+}$and $D_{-}$through the diagram

$$
D_{ \pm}: \Gamma\left(E_{ \pm}\right) \stackrel{\nabla}{\longrightarrow} \Gamma\left(T^{*} M \otimes E_{ \pm}\right) \stackrel{\text { dual }}{\longrightarrow} \Gamma\left(T M \otimes E_{ \pm}\right) \stackrel{\text { cliff }}{\longrightarrow} \Gamma\left(E_{\mp}\right)
$$

where $\nabla$ is the connection on $E=E_{+} \oplus E_{-}$lifted from the Levi-Civita connection on $M$, and cliff denotes the operation of Clifford multiplication. Suppose that we are given an orientation-preserving isometry $T: M \rightarrow M$. Then the tangent map of $T$ gives a map $d T: S O(M) \rightarrow S O(M)$ for which the diagram

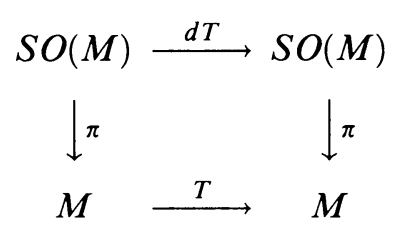

is commutative. Furthermore, $d T$ commutes with the $S O(2 n)$-action. Let $\widetilde{d T}$ be a lifting of $d T$ such that the diagram

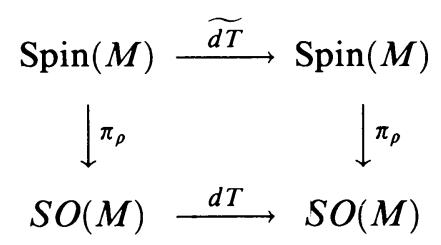

is commutative, and such that $\widetilde{d T}$ commutes with the $\operatorname{Spin}(2 n)$-action. A linear map $T^{*}$ is defined as follows. Suppose that $\phi \in \Gamma\left(E_{ \pm}\right)$is expressed locally over an open set $U \subset M$ by $\phi=[(\sigma, f)]$, where $\sigma: U \rightarrow \operatorname{Spin}(M)$ is a local spin frame field, $f: U \rightarrow \mathscr{S}_{ \pm}$is a spinor-valued function, and $[(\sigma, f)]$ denotes the equivalence class of $(\sigma, f)$ in $E_{ \pm}=\operatorname{Spin}(M) \times_{\rho} \mathscr{S}_{ \pm}$. Let

$$
\left(T^{*} \phi\right)(x)=\left[\left(\widetilde{d T}^{-1} \sigma(x), f\left(T^{-1}\right)\right)\right] .
$$

$T^{*}$ commutes with the Dirac operator $D$, and hence maps $\operatorname{ker} D_{ \pm}$into $\operatorname{ker} D_{ \pm}$. 
Definition 2.1. The Lefschetz number $L(T)$ of the isometry $T$ is defined by

$$
L(T)=\left.\operatorname{Tr} T^{*}\right|_{\text {ker } D_{+}}-\left.\operatorname{Tr} T^{*}\right|_{\text {ker } D_{-}} .
$$

Let $F=\{x \in M \mid T x=x\}$ be the fixed point set of $T . F$ consists of the disjoint union of a finite number of even-dimensional totally geodesic submanifolds $F_{1}, F_{2}, \ldots, F_{r}$. Without loss of generality we assume $r=1$. Let $\nu$ be the normal bundle of $F$ and $\nu(\varepsilon)=\{x \in \nu \mid\|x\|<\varepsilon\}$ for $\varepsilon>0$. The bundle $\nu$ is invariant under $d T$ and $\left.d T\right|_{\nu}$ is nondegenerate.

The Laplacian associated with $D$ is $\Delta=\Delta_{+}+\Delta_{-}$where $\Delta_{ \pm}=D_{\mp} D_{ \pm}$: $\Gamma\left(E_{ \pm}\right) \rightarrow \Gamma\left(E_{ \pm}\right)$. We denote by $P_{t}^{ \pm}(x, y):\left.\left.E_{ \pm}\right|_{y} \rightarrow E_{ \pm}\right|_{x}$ the fundamental solutions for the heat operators $\partial / \partial t+\Delta_{ \pm}$. The standard heat equation argument yields

$$
L(T)=\int_{M}\left(\operatorname{Tr} T^{*} P_{t}^{+}(T x, x)-\operatorname{Tr} T^{*} P_{t}^{-}(T x, x)\right) d x, \quad t>0,
$$

where $d x$ is the Riemannian volume element. Denote the integrand by $\mathscr{L}(t, x)$ $=\operatorname{Tr} T^{*} P_{t}^{+}(T x, x)-\operatorname{Tr} T^{*} P_{t}^{-}(T x, x)$. A routine argument using pseudodifferential operators gives that

$$
|\mathscr{L}(t, x)| \leq C(n, \delta, k) t^{k} \quad \text { as } t \rightarrow 0
$$

for any $k$ in case $d(x, T x) \geq \delta>0$, with $d(\cdot, \cdot)$ denoting the Riemannian distance. Thus

$$
L(T)=\lim _{t \rightarrow 0} \int_{\exp \nu(\varepsilon)} \mathscr{L}(t, x) d x
$$

for any $\varepsilon>0$, and the problem localizes on $F$. Finally, recall the relation

$$
H_{N}(t, x, y)=\frac{\exp \left(\frac{-d(x, y)^{2}}{4 t}\right)}{(4 \pi t)^{n}}(I+O(t))
$$

for the parametrix $H_{N}(t, x, y)$ of $\Delta$ which satisfies

$$
H_{N}(t, x, y)=\frac{\exp \left(\frac{-d^{2}(x, y)}{4 t}\right)}{(4 \pi t)^{n}}\left(\sum_{i=0}^{N} t^{i} U^{(i)}(y, x)\right)
$$

where $N>n$ and $U^{(i)}(y, x): \pi^{-1}(y) \rightarrow \pi^{-1}(x)$ are endomorphisms with $U^{(0)}=$ Id such that for $v \in \pi^{-1}(y)$,

$$
\left(\frac{\partial}{\partial t}+\Delta\right) H_{N}(t, \cdot, y) v=-\frac{\exp \left(\frac{-d^{2}(\cdot, y)}{4 t}\right)}{(4 \pi t)^{n}} t^{N} \Delta U^{(N)}(y, \cdot) v .
$$

Using this, a simple argument working in a local trivialization of $\nu(\varepsilon)$ yields Theorem 2.2. The Lefschetz number $L(T)$ is given by

$$
L(T)=\int_{F} L_{\mathrm{loc}}(T)(\xi) d \xi
$$

where the local Lefschetz number, defined by the limit

$$
L_{\mathrm{loc}}(T)(\xi)=\lim _{t \rightarrow 0} \int_{\nu_{\xi}(\varepsilon)} \mathscr{L}(t, \exp c) d c,
$$

exists and is independent of $\varepsilon$. 
The remaining sections are devoted to evaluating this local index in terms of the geometrical data of the fixed point set and its normal bundle.

\section{ORTHOgONAL AND NORMAL COORDINATES}

Let $y \in M$ and $E(y)=\left(E_{1}(y), \ldots, E_{2 n}(y)\right)$ be an orthonormal frame at $y$. Choose normal coordinates at $y$ and let $E^{y}$ be the orthonormal frame which is parallel along geodesics through $y$ and such that $E^{y}(y)=E(y)$. It is precisely this moving frame with respect to which the parametrix $H_{N}$ for $\Delta$ assumes a tractable form, as described in [9], allowing an evaluation of the local index in the Atiyah-Singer index theorem. However, the action of the isometry $T$ on $S O(M)$ assumes a very simple form near $F$ when we identify a tubular neighborhood of $F$ with a neighborhood of the zero section of $\nu$; that is, in "orthogonal" coordinates. We are thus led to study the relationship between these coordinate systems in this section.

Suppose $F$ is of dimension $2 n^{\prime}$. Let $\xi \in F$ and $E=\left(E_{1}, \ldots, E_{2 n}\right)$ be an oriented orthonormal frame field in a neighborhood of $\xi$ such that

(i) for $\zeta \in F, E_{1}(\zeta), \ldots, E_{2 n^{\prime}}(\zeta)$ are tangent to $F$ while the vector fields $E_{2 n^{\prime}+1}(\zeta), \ldots, E_{2 n}(\zeta)$ are normal to $F$,

(ii) $E$ is parallel along geodesics normal to $F$,

(iii) for $d T$ expressed as a matrix-valued function $\mathscr{T}$ as

$$
d T E(x)=E(T x) \mathscr{T}(x)
$$

then

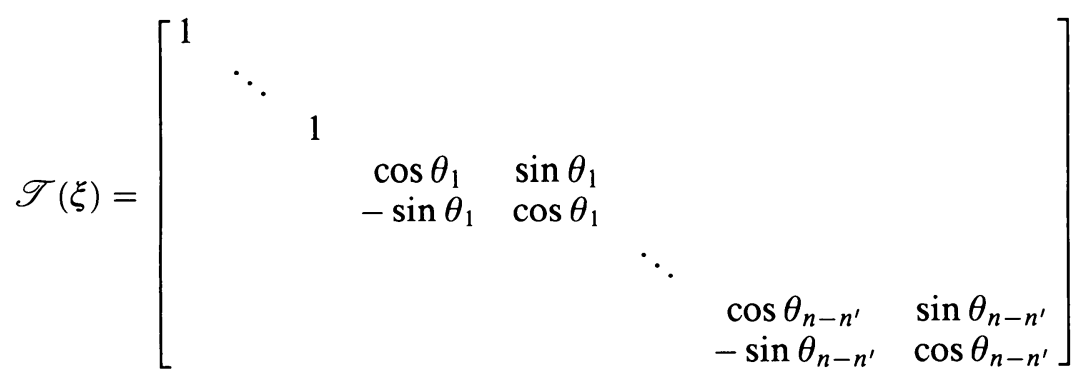

where $0<\theta_{i}<2 \pi$ for $i=1, \ldots, n-n^{\prime}$,

(iv) the orientation of $E$ is that of $M$.

Clearly such a frame field $E$ exists, and moreover there is a neighborhood $V$ of $\xi$ in $F$ such that $E$ is defined on $U=\exp \left(\left.\nu\right|_{V} \cap \nu(\varepsilon)\right)$ for sufficiently small $\varepsilon$. For $B_{0}(\varepsilon)$ the ball of radius $\varepsilon$ in $\mathbf{R}^{2 n-2 n^{\prime}}$ define a homeomorphism $\phi: V \times B_{0}(\varepsilon) \rightarrow U$ by setting

$$
\phi\left(x^{\prime}, c_{1}, \ldots, c_{2 n-2 n^{\prime}}\right)=x=\exp _{x^{\prime}}\left(\sum_{\alpha=1}^{2 n-2 n^{\prime}} c_{\alpha} E_{2 n^{\prime}+\alpha}\left(x^{\prime}\right)\right),
$$

thus identifying $\left(x^{\prime}, c\right)$ with $x=\phi\left(x^{\prime}, c\right)$. Under this identification, Lebesgue measure on the fiber $\nu_{x^{\prime}}$ becomes $d c_{1} \cdots d c_{2 n-2 n^{\prime}}$.

Lemma 3.1. Under the coordinates described by (3.1), the action of $d T$ is constant along fibers of $\nu$; that is, $\mathscr{T}(x)=\mathscr{T}\left(x^{\prime}\right)$ in case $x=\left(x^{\prime}, c\right)$.

Proof. Letting $\gamma(t)=\left(x^{\prime}, t c\right)$ for $0 \leq t \leq 1$, we have that $\gamma(t)$ is a geodesic normal to $F$, and $E\left(x^{\prime}, c\right)=\|_{\gamma} E\left(x^{\prime}\right)$, with $\|_{\gamma}$ denoting parallel transport 
along $\gamma$. Thus,

$$
\begin{aligned}
d T E(x) & =d T\left(\|_{\gamma} E\left(x^{\prime}\right)\right)=\left\|_{T_{\gamma}}\left(d T E\left(x^{\prime}\right)\right)=\right\|_{T_{\gamma}} E\left(x^{\prime}\right) \mathscr{T}\left(x^{\prime}\right) \\
& =\left(u_{T_{\gamma}} E\left(x^{\prime}\right)\right) \mathscr{T}\left(x^{\prime}\right)=E(T x) \mathscr{T}\left(x^{\prime}\right)
\end{aligned}
$$

and hence $\mathscr{T}(x)=\mathscr{T}\left(x^{\prime}\right)$ follows from considering the relation $d T E(x)=$ $E(T x) \mathscr{T}(x)$.

We may now express $\mathscr{T}\left(x^{\prime}\right)$, for $x^{\prime} \in V$, in the form

$$
\mathscr{T}\left(x^{\prime}\right)=\left[\begin{array}{llll}
1 & & & \\
& \ddots & & \\
& & 1 & \\
& & & e^{\Theta\left(x^{\prime}\right)}
\end{array}\right]
$$

where $\Theta\left(x^{\prime}\right) \in \operatorname{so}\left(2 n-2 n^{\prime}\right)$.

Lemma 3.2. Under the homeomorphism (3.1) and the notation of (3.2) the isometry $T$ assumes the form $T\left(x^{\prime}, c\right)=\left(x^{\prime}, c e^{-\Theta\left(x^{\prime}\right)}\right)$.

Proof. Again setting $\gamma(t)=\left(x^{\prime}, t c\right)$ note that $T \gamma$ is a geodesic, and

$$
\begin{aligned}
d T \dot{\gamma}(0) & =d T \sum_{\alpha=1}^{2 n-2 n^{\prime}} c_{\alpha} E_{2 n^{\prime}+\alpha}\left(x^{\prime}\right)=\sum_{\alpha=1}^{2 n-2 n^{\prime}} c_{\alpha} d T E_{2 n^{\prime}+\alpha}\left(x^{\prime}\right) \\
& =\left(E_{2 n^{\prime}+1}\left(x^{\prime}\right), \ldots, E_{2 n}\left(x^{\prime}\right)\right) e^{\Theta\left(x^{\prime}\right)}\left(c_{1}, \ldots, c_{2 n-2 n^{\prime}}\right)^{\top} .
\end{aligned}
$$

Letting $\bar{c}=\left(\bar{c}_{1}, \ldots, \bar{c}_{2 n-2 n^{\prime}}\right)=\left(c_{1}, \ldots, c_{2 n-2 n^{\prime}}\right) e^{-\Theta\left(x^{\prime}\right)}$ we then find that

$$
\begin{aligned}
T\left(x^{\prime}, c\right) & =T\left(\exp _{x^{\prime}} \dot{\gamma}(0)\right)=\exp _{T x^{\prime}} d T \dot{\gamma}(0) \\
& =\exp _{x^{\prime}}\left(\sum_{\alpha=1}^{2 n-2 n^{\prime}} \bar{c}_{\alpha} E_{2 n^{\prime}+\alpha}\left(x^{\prime}\right)\right) \\
& =\left(x^{\prime}, c e^{-\Theta\left(x^{\prime}\right)}\right),
\end{aligned}
$$

completing the proof.

Next consider the oriented orthonormal frame field $E^{T x}$ defined over the patch $U$ by requiring that $E^{T x}(T x)=E(T x)$ and that $E^{T x}$ be parallel along geodesics through $T x$. We define coordinates $y_{i}$ of $x$ by

$$
x=\exp _{T x}\left(\sum_{i=1}^{2 n} y_{i} E_{i}^{T x}(T x)\right),
$$

and a map $\Phi: U \rightarrow s o(2 n)$ by

$$
E^{T x}(x)=E(x) e^{\Phi(x)} .
$$

Lemma 3.3. In case $x=\left(x^{\prime}, c\right)$ then

(i) the coordinates $y_{i}$ satisfy

$$
\begin{aligned}
y_{i} & =o(|c|), & & 1 \leq i \leq 2 n^{\prime}, \\
y_{2 n^{\prime}+\alpha} & =c_{\alpha}-\bar{c}_{\alpha}+o(|c|), & & 1 \leq \alpha \leq 2 n-2 n^{\prime},
\end{aligned}
$$


(ii) the $(i, j)$ element $\Phi_{i j}$ of $\Phi(x)$ satisfies

$$
\Phi_{i j}(x)=-\frac{1}{2} \sum_{\alpha, \beta=1}^{2 n-2 n^{\prime}} \bar{c}_{\alpha} c_{\beta} R_{\alpha \beta i j}\left(x^{\prime}\right)+o\left(|c|^{2}\right)
$$

where

$$
R_{\alpha \beta i j}=-\left\langle\left(\left[\nabla_{E_{2 n^{\prime}+\alpha}}, \nabla_{\left.E_{2 n^{\prime}+\beta}\right]}\right]-\nabla_{\left[E_{2 n^{\prime}+\alpha}, E_{2 n^{\prime}+\beta}\right]}\right) E_{i}, E_{j}\right\rangle .
$$

Proof. The assertion of (i) follows from the relations

$$
y_{i}(x)-y_{i}\left(x^{\prime}\right)=\left(0, \ldots, 0, c_{1}, \ldots, c_{2 n-2 n^{\prime}}\right)+o(|c|)
$$

and

$$
y_{i}\left(x^{\prime}\right)=-\left(0, \ldots, 0, \bar{c}_{1}, \ldots, \bar{c}_{2 n-2 n^{\prime}}\right)
$$

which may be seen from viewing the triangle $\left(T x, x, x^{\prime}\right)$ in the normal coordinates $y_{i}$ at $T x$.

To prove (ii), consider a point $x_{1} \in M$ and vectors $X, Y \in T_{x_{1}} M$. Form a piecewise geodesic path $\left(x_{1}, y_{1}, x_{2}, y_{2}\right)=P$ where

$$
\begin{aligned}
y_{1}=\exp _{x_{1}}(t X), & x_{2}=\exp _{y_{1}}\left(u_{x_{1}}^{y_{1}} t Y\right), \\
y_{2}=\exp _{x_{2}}\left(u_{y_{1}}^{x_{2}} u u_{x_{1}}^{y_{1}}(-t X)\right), & x_{3}=\exp _{y_{2}}\left(l u_{x_{2}}^{y_{2}} u_{y_{1}}^{x_{2}} u_{x_{1}}^{y_{1}}(-t Y)\right)
\end{aligned}
$$

with $l l_{x}^{y}$ denoting parallel translation from $x$ to $y$. Then $x_{3}=x_{1}+o\left(t^{2}\right)$ and parallel translation of a vector $Z \in T_{x_{1}}$ around $P$ results in a vector

$$
u_{P} Z=Z+t^{2} R(X, Y) Z+o\left(t^{2}\right) .
$$

Now, since $u_{x_{1}}^{y_{1}} t Y=t Y+o(t)$ and $u_{y_{1}}^{x_{2}} u_{x^{1}}^{y^{1}}(-t X)=-t X+o(t)$ we may think of the triangles $T_{1}=\left(x_{1}, y_{1}, y_{2}\right)$ and $T_{2}=\left(x_{2}, y_{2}, y_{1}\right)$ as being similar to order $o(t)$ and then find that

$$
u_{T_{1}} Z=Z+\frac{t^{2}}{2} R(X, Y) Z+o\left(t^{2}\right) .
$$

Applying this argument to the vectors

$$
\left(0, \ldots, 0, \bar{c}_{1}, \ldots, \bar{c}_{2 n-2 n^{\prime}}\right)
$$

and

$$
\left(0, \ldots, 0, c_{1}, \ldots, c_{2 n-2 n^{\prime}}\right)
$$

at $x^{\prime}$ yields the conclusion of (ii).

The frames $E^{T x}$ and $E$ are thus related by an infinitesimal holonomy.

\section{The Clifford ASYMptotics}

Choose a spin frame field $\sigma: U \rightarrow \operatorname{Spin}(M)$ such that

$$
\pi_{\rho} \sigma=\left(E_{1}^{T x}, \ldots, E_{2 n}^{T x}\right) .
$$

For $x \in U$, let $\bar{P}_{t}(x), \bar{T}^{*}(x) \in \operatorname{Hom}\left(\mathscr{S}_{ \pm}, \mathscr{S}_{ \pm}\right)$be defined through the equivalence relations

$$
P_{t}(T x, x)[(\sigma(x), v)]=\left[\left(\sigma(T x), \bar{P}_{t}(x) v\right)\right]
$$

and

$$
T^{*}[(\sigma(T x), u)]=\left[\left(\sigma(x), \bar{T}^{*}(x) u\right)\right] .
$$

Then we easily obtain 
Lemma 4.1. For $x$ in a sufficiently small neighborhood of $F$ and $t>0$, the integrand $\mathscr{L}(t, x)$ is evaluated by

$$
\mathscr{L}(t, x)=\left.\operatorname{Tr} \bar{T}^{*}(x) \bar{P}_{t}(x)\right|_{\mathscr{S}_{+}}-\left.\operatorname{Tr} \bar{T}^{*}(x) \bar{P}_{t}(x)\right|_{\mathscr{S}_{-}}=\operatorname{Tr}_{s} \bar{T}^{*} \bar{P}_{t}(x)
$$

with the second equality defining the supertrace $\operatorname{Tr}_{s}$.

In [9] there is an operator $\chi$ defined on monomials in the Clifford variables which is precisely the bookkeeping device needed for a direct evaluation of the asymptotics of the heat kernel trace. In the normal coordinates $y_{1}, \ldots, y_{2 n}$ this may be defined as

$$
\chi\left(y^{\alpha} D_{y}^{\beta} e^{\gamma}\right)=|\beta|-|\alpha|+|\gamma|
$$

for multi-indices $\alpha, \beta$ and $\gamma$ with

$$
y^{\alpha}=y_{1}^{\alpha_{1}} \cdots y_{2 n}^{\alpha_{2 n}},
$$

and

$$
D_{y}^{\beta}=\left(\partial / \partial y_{1}\right)^{\beta_{1}} \cdots\left(\partial / \partial y_{2 n}\right)^{\beta_{2 n}}
$$

and $e^{\gamma}=e_{1}^{\gamma_{1}} \cdots e_{2 n}^{\gamma_{2 n}}$ for $\gamma_{i} \in \mathbf{Z}_{2}$. For our purposes here, a less refined analysis will suffice. We first rescale the metric in normal directions, setting $x=\left(x^{\prime}, c\right)$ with $x^{\prime}=\xi \in F$ and $c=\sqrt{t b}$ with $b$ and $\xi$ fixed. Now, for a monomial $\phi(t) e_{i_{1}} \cdots e_{i_{s}}$ with $\phi(t) \in \mathbf{R}$ define a modified $\chi$ operator by setting

$$
\bar{\chi}\left(\phi(t) e_{i_{1}} \cdots e_{i_{s}}\right)=s-\sup \left\{k \in \mathbf{Z}\left|\lim _{t \rightarrow 0^{+}} \frac{|\phi(t)|}{t^{k / 2}}\right|<\infty\right\} .
$$

We will write $P=Q+(\bar{\chi}<m)$ to denote congruence modulo the space of monoids with $\bar{\chi}<m$; that is, in case $\bar{\chi}(P-Q)<m$.

Considering Lemma 3.1 we see that $d T E(x)=E(T x) \mathscr{T}(\xi)$, and

$$
\begin{aligned}
d T E^{T(x)} & =d T\left(E(x) e^{\Phi(x)}\right)=E(T x) \mathscr{T}(\xi) e^{\Phi(x)} \\
& =E^{T x}(T x) \mathscr{T}(\xi) e^{\Phi(x)}=E^{T x} e^{\Psi(\xi)} e^{\Phi(x)}
\end{aligned}
$$

since $T$ is an isometry, where

$$
\Psi=\left[\begin{array}{llll}
0 & & & \\
& \ddots & & \\
& & 0 & \\
& & & \boldsymbol{\Theta}(\xi)
\end{array}\right],
$$

and

$$
\boldsymbol{\Theta}(\xi)=\left[\begin{array}{ccccc}
0 & \theta_{1} & & & \\
-\theta_{1} & 0 & & & \\
& & \ddots & & \\
& & & 0 & \theta_{n-n^{\prime}} \\
& & & -\theta_{n-n^{\prime}} & 0
\end{array}\right] .
$$

Thus,

$$
\begin{aligned}
\widetilde{d T} \sigma(x)= & \sigma(T x) \exp \left(-\frac{1}{2} \sum_{\alpha=1}^{n-n^{\prime}} \theta_{\alpha} e_{2 n^{\prime}+2 \alpha-1} e_{2 n^{\prime}+2 \alpha}\right) \\
& \times \exp \left(-\frac{1}{2} \sum_{1 \leq i, j \leq 2 n} \Phi_{i j}(x) e_{i} e_{j}\right)
\end{aligned}
$$


and then

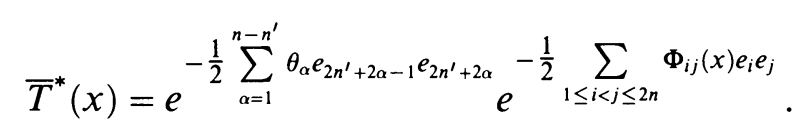

Computing the exponential for $\operatorname{Spin}(2 n)$ then gives

$$
\begin{aligned}
\exp & \left(-\frac{1}{2} \sum_{\alpha=1}^{n-n^{\prime}} \theta_{\alpha} e_{2 n^{\prime}+2 \alpha-1} e_{2 n^{\prime}+2 \alpha}\right) \\
& =\prod_{\alpha=1}^{n-n^{\prime}}\left(\cos \frac{\theta_{\alpha}}{2}-\sin \frac{\theta_{\alpha}}{2} e_{2 n^{\prime}+2 \alpha-1} e_{2 n^{\prime}+2 \alpha}\right) \\
& =(-1)^{n-n^{\prime}}\left(\prod_{\alpha=1}^{n-n^{\prime}} \sin \frac{\theta_{\alpha}}{2} e_{2 n^{\prime}+1} e_{2 n^{\prime}+2} \cdots e_{2 n}\right)+\left(\bar{\chi}<2\left(n-n^{\prime}\right)\right) .
\end{aligned}
$$

Let $A^{\perp}$ be the $\left(2 n-2 n^{\prime}\right) \times\left(2 n-2 n^{\prime}\right)$ matrix whose $(\alpha, \beta)$ element is given by

$$
\left(A^{\perp}\right)_{\alpha \beta}=-\frac{1}{2} \sum_{i, j=1}^{2 n^{\prime}} R_{\alpha \beta i j}(\xi) e_{i} e_{j} .
$$

$A^{\top}$ is defined in the obvious and analogous fashion, replacing $\alpha$ and $\beta$ by indices ranging between 1 and $2 n^{\prime}$, accounting for the tangential directions. Lemma 3.3 yields then that

$$
\begin{aligned}
\exp \left\{-\frac{1}{2} \sum_{1 \leq i<j \leq 2 n} \Phi_{i j}(x) e_{i} e_{j}\right\}=\exp \left[-\frac{t}{4} \sum_{\alpha, \beta=1}^{2 n-2 n^{\prime}} b_{\alpha} b_{\beta}\left(e^{-\Theta(\xi)} A^{\perp}\right)_{\alpha \beta}\right. \\
\left.+\sum_{\alpha=1}^{2 n-2 n^{\prime}} e_{2 n+\alpha}(\bar{\chi}<0)+(\bar{\chi}<0)\right] .
\end{aligned}
$$

Summarizing, the above observations prove the following

Lemma 4.2. The operator $\bar{T}^{*}$ is given by

$$
\begin{aligned}
\bar{T}^{*}(x)= & (-1)^{n-n^{\prime}}\left(\prod_{\alpha=1}^{n-n^{\prime}} \sin \frac{\theta_{\alpha}}{2}\right) \exp \left[-\frac{t}{4} \sum_{\alpha, \beta=1}^{2 n-2 n^{\prime}} b_{\alpha} b_{\beta}\left(e^{-\Theta(\xi)} A^{\perp}\right)_{\alpha \beta}\right] \\
& \times e_{2 n^{\prime}+1} \cdots e_{2 n}+\left(\bar{\chi}<2\left(n-n^{\prime}\right)\right) .
\end{aligned}
$$

Finally, let $\tilde{A}$ be the $2 n \times 2 n$ matrix given by

$$
\tilde{A}_{i j}=-\frac{1}{2} \sum_{k, l=1}^{2 n} R_{i j k l}^{T x} e_{k} e_{l},
$$

where $R_{i j k l}^{T x}$ are the components of the Riemannian curvature tensor with respect to the frame field $E^{T x}$, and set

$$
\widetilde{A}^{k}(y)=\sum_{i, j=1}^{2 n} y_{i} y_{j}\left(\widetilde{A}^{k}\right)_{i j}, \quad k=1,2, \ldots
$$


Now, from [9] we infer that there is $P\left(t ; z_{1}, z_{2}, \ldots ; w_{1}, w_{2}, \ldots\right)$, an operator which is a power series in $t$ with coefficient polynomials in $z_{i}$ and $w_{i}$ such that

$$
\begin{aligned}
\bar{P}_{t}(x)= & \left(\frac{1}{4 \pi t}\right)^{n} \exp \left(\frac{-d^{2}(x, T x)}{4 t}\right) \\
& \times\left[P \left(t ; \operatorname{Tr} \widetilde{A}^{2}, \ldots, \operatorname{Tr} \widetilde{A}^{2 k}, \ldots, \operatorname{Tr} \widetilde{A}^{2 n} ;\right.\right. \\
& \left.\left.\widetilde{A}^{2}(y), \ldots, \widetilde{A}^{2 k}(y), \ldots, \widetilde{A}^{2 n}(y)\right)+\sum_{m \geq 0} t^{m}(\bar{\chi}<2 m)\right],
\end{aligned}
$$

where in diagonal form we have, by solving harmonic oscillator-type equations,

$$
\begin{aligned}
& P(t\left.;\left((-1)^{k} 2\left(u_{1}^{2 k}+\cdots+u_{n}^{2 k}\right)\right) ;\left((-1)^{k} \sum_{\alpha=1}^{n}\left(v_{2 \alpha-1}^{2}+v_{2 \alpha}^{2}\right) u_{\alpha}^{2 k}\right)\right) \\
& \quad=(4 \pi t)^{n} e^{\frac{\|v\|^{2}}{4 t}} \prod_{\alpha=1}^{n}\left(\frac{i u_{\alpha}}{8 \pi \sinh \frac{i u_{\alpha} t}{2}} \exp \left(\frac{-i u_{\alpha}}{8}\left(v_{2 \alpha-1}^{2}+v_{2 \alpha}^{2}\right) \operatorname{coth} \frac{i u_{\alpha} t}{2}\right)\right) .
\end{aligned}
$$

Lemma 4.3. Let $\tilde{A_{0}}$ be the matrix

$$
\left(\widetilde{A_{0}}\right)_{i j}=-\frac{1}{2} \sum_{k, l=1}^{2 n^{\prime}} R_{i j k l}(\xi) e_{k} e_{l}, \quad 1 \leq i, j \leq 2 n,
$$

and define the tangential component $A^{\top}$ by

$$
\left(\tilde{A}^{\top}\right)_{i j}=-\frac{1}{2} \sum_{k, l=1}^{2 n^{\prime}} R_{i j k l}(\xi) e_{k} e_{l}, \quad 1 \leq i, j \leq 2 n^{\prime} .
$$

Then

$$
\tilde{A_{0}}=\left(\begin{array}{cc}
A^{\top} & 0 \\
0 & A^{\perp}
\end{array}\right)
$$

Furthermore, the relations

$$
\operatorname{Tr} \widetilde{A}^{2 k}=\operatorname{Tr}\left(A^{\top}\right)^{2 k}+\operatorname{Tr}\left(A^{\perp}\right)^{2 k}+\sum_{\alpha=1}^{2\left(n-n^{\prime}\right)} e_{2 n^{\prime}+\alpha}(\bar{\chi}<4 k)
$$

and

$$
\widetilde{A}^{2 k}(y / \sqrt{t})=\left(A^{\perp}\right)^{2 k}(b-\bar{b})+\sum_{\alpha=1}^{2\left(n-n^{\prime}\right)} e_{2 n^{\prime}+\alpha}(\bar{\chi}<4 k)
$$

hold.

Proof. Let $R_{\alpha}=R_{2 n^{\prime}+\alpha, i j k}(\xi)$ for $1 \leq i, j, k \leq 2 n^{\prime}, 1 \leq \alpha \leq 2\left(n-n^{\prime}\right)$. Then

$$
\begin{aligned}
R_{\alpha} & =-\left\langle R\left(E_{2 n^{\prime}+\alpha}(\xi), E_{i}(\xi)\right) E_{j}(\xi), E_{k}(\xi)\right\rangle \\
& =-\left\langle R\left(d T E_{2 n^{\prime}+\alpha}(\xi), d T E_{i}(\xi)\right) d T E_{j}(\xi), d T E_{k}(\xi)\right\rangle \\
& =-\left\langle R\left(d T E_{2 n^{\prime}+\alpha}(\xi), E_{i}(\xi)\right) E_{j}(\xi), E_{k}(\xi)\right\rangle
\end{aligned}
$$


and thus

$$
\left(R_{1}, \ldots, R_{2\left(n-n^{\prime}\right)}\right)=\left(R_{1}, \ldots, R_{2\left(n-n^{\prime}\right)}\right) e^{\Theta(\xi)} .
$$

It follows that

$$
\left(R_{1}, \ldots, R_{2\left(n-n^{\prime}\right)}\right)=0
$$

since $d T$ is nondegenerate and $e^{\boldsymbol{\theta}}$ does not have 1 as an eigenvalue. This proves (4.2). The relations (4.3) and (4.4) now follow from (4.2), Lemma 3.3, and the facts

$$
\operatorname{Tr} \widetilde{A}^{2 k}=\operatorname{Tr} \tilde{A}_{0}^{2 k}+\sum_{\alpha=1}^{2\left(n-n^{\prime}\right)} e_{2 n^{\prime}+\alpha}(\bar{\chi}<4 k)
$$

and

$$
\tilde{A}^{2 k}(y / \sqrt{t})=\tilde{A}_{0}^{2 k}(b-\bar{b})+\sum_{\alpha=1}^{2\left(n-n^{\prime}\right)} e_{2 n^{\prime}+\alpha}(\bar{\chi}<4 k),
$$

which complete the proof.

We may now summarize the results of this section in

Lemma 4.4. The Lefschetz integrand $\mathscr{L}(t, x)$ is given by (4.1), where

$$
\bar{T}^{*}(x) \bar{P}_{t}(x)=e^{\frac{-\|b-\bar{b}\|^{2}}{4}}\left[\frac{(-1)^{n-n^{\prime}}}{(4 \pi t)^{n}}\left(\prod_{\alpha=1}^{n-n^{\prime}} \sin \frac{\theta_{\alpha}}{2}\right)\right.
$$

$$
\begin{aligned}
& \times \exp \left(\frac{-t}{4} \sum_{\alpha, \beta=1}^{2\left(n-n^{\prime}\right)} b_{\alpha} b_{\beta}\left(e^{-\Theta(\xi)} A^{\perp}\right)_{\alpha \beta}\right) \\
& \times P\left(t ;\left(\operatorname{Tr}\left(A^{\top}\right)^{2 k}+\operatorname{Tr}\left(A^{\perp}\right)^{2 k}\right) ;\left(t\left(A^{\perp}\right)^{2 k}(b-\bar{b})\right)\right) \\
& \left.\times e_{2 n^{\prime}+1} \cdots e_{2 n}+e^{\sqrt{t} f}\left(\bar{\chi}<4 n-2 n^{\prime}\right)_{b}\right] .
\end{aligned}
$$

Here $f$ is a bounded and continuous function, and $\left(\bar{\chi}<4 n-2 n^{\prime}\right)_{b}$ denotes the space spanned by monoids which are polynomials in $b$, and satisfy $\bar{\chi}<$ $4 n-2 n^{\prime}$.

\section{EVAluation OF THE LOCAL INDEX}

We begin by showing that $L_{\text {loc }}$ depends only on the first term in (4.5).

Lemma 5.1. Let $\phi$ be an arbitrary element of $\left(\bar{\chi}<4 n-2 n^{\prime}\right)_{b}$. Then

$$
\lim _{t \rightarrow 0} \int_{\mathbf{R}^{2 \mathbf{n}-2 \mathbf{n}^{\prime}}} t^{n-n^{\prime}} e^{\frac{-\|b-\bar{b}\|^{2}}{4}} \operatorname{Tr}_{s} \phi d b=0 .
$$

Proof. We may assume that $\phi=b_{i_{1}} \cdots b_{i_{m}} \psi$ where $\psi$ is independent of $b$ and satisfies $\bar{\chi}\left(t^{n-n^{\prime}} \psi\right)<2 n$. Then the simple observations that

$$
\left|\int_{\mathbf{R}^{2 \mathbf{n}-2 \mathbf{n}^{\prime}}} e^{\frac{-\|b-\bar{b}\|^{2}}{4}} b_{i_{1}} \cdots b_{i_{m}} d b\right|<\infty
$$


and

$$
\lim _{t \rightarrow 0^{+}} t^{n-n^{\prime}} \operatorname{Tr}_{s} \phi=0
$$

together give the result of the lemma.

To compute the supertrace it thus suffices to compute the coefficient of the $e_{1} \cdots e_{2 n}$ term in (4.5). Note that $A^{\perp}$ and $A^{\top}$ are of order $\bar{\chi}=2$, containing terms $e_{i} e_{j}$ with $1 \leq i, j \leq 2 n^{\prime}$. Note also that $e_{i} e_{j}=-e_{j} e_{i}+(\bar{\chi}<1)$. Thus, if we formally replace $e_{i}$ by $\omega_{i}$, where $\omega=\left(\omega_{1}, \ldots, \omega_{2 n}\right)$ is the frame dual to $E$, and then substitute $\Omega^{\top}$ and $\Omega^{\perp}$ for $A^{\top}$ and $A^{\perp}$, where

$$
\begin{aligned}
& \Omega^{\top}=-\frac{1}{2} \sum_{k, l=1}^{2 n} R_{i j k l} \omega_{k} \wedge \omega_{l}, \quad 1 \leq i, j \leq 2 n^{\prime}, \\
& \Omega^{\perp}=-\frac{1}{2} \sum_{k, l=1}^{2 n} R_{i j k l} \omega_{k} \wedge \omega_{l}, \quad 2 n^{\prime}+1 \leq i, j \leq 2 n,
\end{aligned}
$$

then computing the supertrace is equivalent to computing the form of the top order $2 n^{\prime}$ on $F$, if we multiply by $(2 / \sqrt{-1})^{n}$, which is the so-called BerezinPatodi constant. (To explain the appearance of this term, simply note that $\left.\operatorname{Tr}\right|_{\mathscr{S}_{ \pm}}\left(e_{1} \cdots e_{2 n}\right)=2^{n-1} / \sqrt{-1}^{n}$.) It remains then to compute this differential form, and to evaluate the $t \rightarrow 0$ limit.

Let

$$
\boldsymbol{\Omega}=\left[\begin{array}{cc}
\Omega^{\top} & 0 \\
0 & \Omega^{\perp}
\end{array}\right]
$$

be given formally as

$$
\Omega^{\top}=\left[\begin{array}{ccccc}
0 & u_{1} & & & \\
-u_{1} & 0 & & & \\
& & \ddots & & \\
& & & 0 & u_{n^{\prime}} \\
& & & -u_{n^{\prime}} & 0
\end{array}\right], \quad \Omega^{\perp}=\left[\begin{array}{ccccc}
0 & v_{1} & & & \\
-v_{1} & 0 & & & \\
& & \ddots & & \\
& & 0 & v_{n^{\prime}} \\
& & -v_{n^{\prime}} & 0
\end{array}\right],
$$

where $v_{i}$ and $u_{i}$ are indeterminants. Then

$$
\begin{aligned}
& \sum_{\alpha, \beta=1}^{2\left(n-n^{\prime}\right)} b_{\alpha} b_{\beta}\left(\exp ^{-\Theta(\xi)} \Omega^{\perp}\right)_{\alpha \beta}=\sum_{\alpha=1}^{n-n^{\prime}} \sin \theta_{\alpha} \cdot v_{\alpha}\left(b_{2 \alpha-1}^{2}+b_{2 \alpha}^{2}\right), \\
& \Omega^{2 k}(y)=(-1)^{k} \sum_{\alpha=1}^{n-n^{\prime}} 4 t \sin ^{2} \frac{\theta_{\alpha}}{2} v_{\alpha}^{2 k}\left(b_{2 \alpha-1}^{2}+b_{2 \alpha}^{2}\right), \\
& \operatorname{Tr} \Omega^{2 k}=2(-1)^{k}\left(\sum_{\alpha} u_{\alpha}^{2}+\sum_{\beta} v_{\beta}^{2}\right) .
\end{aligned}
$$


The above lemmas then give that as a $2 n^{\prime}$ form on $F$,

$$
\begin{aligned}
L_{\mathrm{loc}}(T) & =\lim _{t \rightarrow 0} \int_{\mathbf{R}^{2 n-2 n^{\prime}}}(-1)^{n-n^{\prime}}\left(\frac{2}{\sqrt{-1}}\right)^{n} t^{n-n^{\prime}} \\
& \times \prod_{\alpha=1}^{n-n^{\prime}} \sin \frac{\theta_{\alpha}}{2}\left(\frac{1}{4 \pi t}\right)^{n} \prod_{\alpha=1}^{n^{\prime}} \frac{i t u_{\alpha} / 2}{\sinh i t u_{\alpha} / 2} \\
& \times \exp \left(\frac{-t}{4} \sum_{\alpha=1}^{n-n^{\prime}} \sin \theta_{\alpha} \cdot v_{\alpha}\left(b_{2 \alpha-1}^{2}+b_{2 \alpha}^{2}\right)\right) \prod_{\beta=1}^{n-n^{\prime}} \frac{i t v_{\beta} / 2}{\sinh i t v_{\beta} / 2} \\
& \times \exp \left(\sum_{\alpha=1}^{n-n^{\prime}}\left(\frac{-\sqrt{-1}}{2} v_{\alpha} t \sin ^{2} \frac{\theta_{\alpha}}{2} \operatorname{coth} \sqrt{-1} \frac{v_{\alpha} t}{2}\left(b_{2 \alpha-1}^{2}+b_{2 \alpha}^{2}\right)\right)\right) d b .
\end{aligned}
$$

Now in the final calculation, after integrating out $b$, we will take the form of order $2 n^{\prime}$ on $F$, and hence the factor of $t$ cancels. We are left then with the evaluation

$$
\begin{aligned}
L_{\mathrm{loc}}(T)= & (-1)^{n-n^{\prime}}\left(\frac{1}{2 \pi \sqrt{-1}}\right)^{n} \int_{\mathbf{R}^{2 n-2 n^{\prime}}} \prod_{\alpha=1}^{n-n^{\prime}} \sin \frac{\theta_{\alpha}}{2} \\
& \times \prod_{\gamma=1}^{n^{\prime}} \frac{\sqrt{-1} u_{\gamma} / 2}{\sinh \sqrt{-1} u_{\gamma} / 2} \prod_{\beta=1}^{n-n^{\prime}} \frac{\sqrt{-1} v_{\beta} / 2}{\sinh \sqrt{-1} v_{\beta} / 2} \\
& \times \exp \left(\sum_{\gamma}\left(-\frac{1}{4} v_{\gamma} \sin \theta_{\gamma}-\frac{\sqrt{-1}}{2} v_{\gamma} \operatorname{coth} \frac{\sqrt{-1} v_{\gamma}}{2} \sin ^{2} \frac{\theta_{\gamma}}{2}\right)\right. \\
= & (-1)^{n-n^{\prime}}\left(\frac{1}{2 \pi \sqrt{-1}}\right)^{n} \int_{\mathbf{R}^{2 n-2 n^{\prime}}}^{2} \prod_{\alpha=1}^{n-n^{\prime}} \sin \frac{\theta_{\alpha}}{2} \\
& \times \prod_{\beta=1}^{n^{\prime}} \frac{\sqrt{-1} u_{\gamma} / 2}{\sinh \sqrt{-1} u_{\gamma} / 2} \prod_{\beta=1}^{n-n^{\prime}} \frac{\sqrt{-1} v_{\beta} / 2}{\sinh \sqrt{-1} v_{\beta} / 2} d b \\
& \times \exp \left(-\frac{1}{2} \sum_{\gamma} \frac{v_{\gamma} \sin \theta_{\gamma} / 2}{\sin v_{\gamma} / 2} \sin \left(\frac{v_{\gamma}+\theta_{\gamma}}{2}\right)\left(b_{2 \gamma-1}^{2}+b_{2 \gamma}^{2}\right)\right) d b \\
= & (-1)^{n-n^{\prime}}\left(\frac{1}{2 \pi \sqrt{-1}}\right)^{n} \pi^{n-n^{\prime}} \prod_{\alpha=1}^{n^{\prime}} \frac{\sqrt{-1} u_{\alpha} / 2}{\sinh \sqrt{-1} u_{\alpha} / 2} \prod_{\beta=1}^{n-n^{\prime}} \sin \left(\frac{v_{\beta}}{2}+\frac{\theta_{\beta}}{2}\right) \\
= & \prod_{\alpha=1}^{n^{\prime}} \frac{u_{\alpha}^{*} / 2 \pi}{\sinh u_{\alpha}^{*} / 2 \pi}\left(\prod_{\beta=1}^{n-n^{\prime}} 2 \sinh \left(\frac{v_{\beta}^{*}}{2 \pi}+\frac{\sqrt{-1} \theta_{\beta}}{2}\right)\right)
\end{aligned}
$$

where we make use of the Chern roots $u_{i}^{*}$ and $v_{i}^{*}$ expressed as

$$
\operatorname{det}\left(1+\frac{\Omega^{\top}}{2 \pi}\right)=\prod_{i=1}^{n^{\prime}}\left(1+\left(u_{i}^{*}\right)^{2}\right), \quad \operatorname{det}\left(1+\frac{\Omega^{\perp}}{2 \pi}\right)=\prod_{j=1}^{n-n^{\prime}}\left(1+\left(v_{j}^{*}\right)^{2}\right) .
$$


We thus obtain the main result

Theorem 5.2. The Lefschetz number $L(T)$ of the isometry $T$ acting on the spin manifold $M$ is expressed by

$$
L(T)=\sum_{i} \int_{F_{i}}\left[L_{\mathrm{loc}}(T)\right]_{i}
$$

where in the notation used above

$$
\begin{aligned}
{\left[L_{\mathrm{loc}}(T)\right]_{i} } & =\sqrt{\operatorname{det} \frac{\Omega^{\top} / 4 \pi}{\sin \Omega^{\top} / 4 \pi}} \operatorname{Pf}\left(2 \sin \left(\Omega^{\perp} / 4 \pi+\sqrt{-1} \Theta / 2\right)\right)^{-1} \\
& =\widehat{A}\left(T F_{i}\right)\left[\operatorname{Pf}(2 \sin (\Omega / 4 \pi+\sqrt{-1} \Theta / 2))\left(\nu\left(F_{i}\right)\right)\right]^{-1}
\end{aligned}
$$

It is a simple matter to extend the above procedure to the case of a twisted spin complex.

\section{ACKNOWLEDGMENTS}

We are grateful to a referee, who pointed out a related result [6] in the supersymmetric physics literature. Finally, the first author would like to express his gratitude to Professor S. S. Chern for his generous invitation to visit the Nankai Institute of Mathematics.

\section{REFERENCES}

1. M. F. Atiyah and R. Bott, $A$ Lefschetz fixed point formula for elliptic complexes. I, Ann. of Math. (2) 86 (1967), 374-407.

2. __ A Lefschetz fixed point formula for elliptic complexes. II, Ann. of Math. (2) 88 (1968), 451-491.

3. N. Berline and M. Vergne, A computation of the equivariant index of the Dirac operator, Bull. Soc. Math. France 113 (1985), 305-345.

4. J. M. Bismut, The Atiyah-Singer theorems: a probabilistic approach II. The Lefschetz fixed point formulas, J. Funct. Anal. 57 (1984), 329-348.

5. E. Getzler, A short proof of the local Atiyah-Singer index theorem, Topology 25 (1) (1986), 111-117.

6. M. W. Goodman, Proof of character-valued index theorems, Comm. Math. Phys. 107 (1986), 391-409.

7. V. K. Patodi, An analytic proof of the Riemann-Roch-Hirzebruch theorem on Kaehler manifolds, J. Differential Geom. 5 (1971), 251-283.

8. $\_$, Curvature and the eigenforms of the Laplace operator, J. Differential Geom. 5 (1971), 233-249.

9. Y. L. Yu, Local index theorem for Dirac operator, Acta Math. Sinica (N.S.) 3 (2) (1987), 152-169.

10. W. P. Zhang, The local Atiyah-Singer index theorem for families of Dirac operators, Lecture Notes in Math., vol. 1369, Springer-Verlag, Berlin and New York, 1989, pp. 351-366.

ibM Thomas J. Watson Research Center, Yorktown Heights, New York 10598

Academia Sinica, Beijing, People's Republic of China

UNIVERSite PARIS-SUd, 91405 OrSAy, France 